\title{
More general viscosity implicit midpoint rule for nonexpansive mapping with applications
}

\author{
Hui-Ying $\mathrm{Hu}$ \\ Department of Mathematics, Shanghai Normal University, Shanghai, 200234, China.
}

Communicated by C. Vetro

\begin{abstract}
The more general viscosity implicit midpoint rule of fixed point of nonexpansive mapping in Hilbert space is established. The strong convergence of this rule is proved under certain assumptions imposed on the sequence of parameters, which, in addition, is the unique solution of the variational inequality problem. Applications to variational inequalities, hierarchical minimization problems, Fredholm integral equations, and nonlinear evolution equations are included. The results presented in this work may be treated as an improvement, extension and refinement of some corresponding ones in the literature. (C)2017 All rights reserved.
\end{abstract}

Keywords: More general viscosity implicit midpoint rule, nonexpansive mapping, fixed point problem, iterative scheme, variational inequality.

2010 MSC: 47J25, 47N20, 34G20, 65J15.

\section{Introduction}

Throughout the paper unless otherwise stated, $\mathrm{H}$ denotes a real Hilbert space, we denote the norm and inner product of $\mathrm{H}$ by $\|\cdot\|$ and $\langle\cdot, \cdot\rangle$, respectively. Let $C$ be a nonempty, closed and convex subset of $H$. Let $\left\{x_{n}\right\}$ be a sequence in $H$, then $x_{n} \rightarrow x$ (respectively, $x_{n} \rightarrow x$ ) will denote strong (respectively, weak) convergence of the sequence $\left\{x_{n}\right\}$.

The viscosity approximation method for nonexpansive mappings in Hilbert spaces was introduced by Moudafi [12], following the ideas of Attouch [2], which generates the sequence $\left\{x_{n}\right\}$ by the following explicit viscosity method:

$$
x_{n+1}=\alpha_{n} f\left(x_{n}\right)+\left(1-\alpha_{n}\right) T x_{n}, \quad n \geqslant 0,
$$

where $f$ is a contraction mapping on $H, T$ is a nonexpansive mapping on $H$, and $\left\{\alpha_{n}\right\}$ is a sequence in $(0,1)$. Note that the iterative scheme (1.1) generalizes the results of Browder [6] and Halpern [9] in another direction. The convergence of the explicit iterative scheme (1.1) has been the subject of many authors because under suitable conditions these iterations converge strongly to the unique solution $q \in \operatorname{Fix}(T)$ of the variational inequality (VI)

$$
\langle(I-f) q, x-q\rangle \geqslant 0, \quad x \in \operatorname{Fix}(T),
$$

Email address: huiying1117@hotmail. com (Hui-Ying $\mathrm{Hu}$ )

doi:10.22436/jnsa.010.05.41 
where Fix $(T)$ denotes the set of fixed points of $T$. This fact allows us to apply this method to convex optimization, linear programming, and monotone inclusions. In 2004, $\mathrm{Xu}$ [17] extended the result of Moudafi [12] to uniformly smooth Banach spaces and obtained strong convergence theorem.

In 2006, Marino and Xu [11] introduced the following iterative scheme based on viscosity approximation method, for fixed point problem for nonexpansive mapping $\mathrm{T}$ on $\mathrm{H}$ :

$$
x_{n+1}=\alpha_{n} \gamma f\left(x_{n}\right)+\left(I-\alpha_{n} B\right) T x_{n}, \quad n \geqslant 0,
$$

where $f$ is a contraction mapping on $H, B$ is a strongly positive self-adjoint bounded linear operator on $H$, and $T$ is a nonexpansive mapping on $H$. They proved that sequence $\left\{x_{n}\right\}$ generated by (1.3) converges strongly to the unique solution of the variation inequality (VI)

$$
\langle(B-\gamma f) q, x-q\rangle \geqslant 0, \quad x \in \operatorname{Fix}(T),
$$

which is the optimality condition for minimization problem

$$
\min _{x \in \operatorname{Fix}(T)} \frac{1}{2}\langle B x, x\rangle-h(x),
$$

where $h$ is the potential function for $\gamma f$.

The implicit midpoint rule (IMR) is one of the powerful methods for solving ordinary differential equation and differential algebraic equations, see $[3,4,8,14-16]$ and the references therein. For instance, consider the initial value problem for differential equation $y^{\prime}(t)=f(y(t))$ with the initial condition $y(0)=$ $y_{0}$, where $f$ is a continuous function form $R^{d}$ to $R^{d}$. The IMR is an implicit method that generates a sequence $\left\{y_{n}\right\}$ via the relation

$$
\frac{1}{h}\left(y_{n+1}-y_{n}\right)=f\left(\frac{y_{n+1}+y_{n}}{2}\right) .
$$

In 2014, IMR has been extended by Alghamdi et al. [1] to nonexpansive mappings, which generates a sequence $\left\{x_{n}\right\}$ by the following implicit iterative scheme:

$$
x_{n+1}=\alpha_{n} x_{n}+\left(1-\alpha_{n}\right) T\left(\frac{x_{n}+x_{n+1}}{2}\right), n \geqslant 0,
$$

where the initial guess $x_{0} \in H$ is arbitrarily chosen, and $\alpha_{n} \in(0,1)$ for all $n$.

In 2015, Xu et al. [19] extended and generalized the results of Alghamdi et al. [1] and presented the following viscosity implicit midpoint rule (VIMR, for short):

$$
x_{n+1}=\alpha_{n} f\left(x_{n}\right)+\left(1-\alpha_{n}\right) T\left(\frac{x_{n}+x_{n+1}}{2}\right), n \geqslant 0,
$$

where $f$ is a contraction mapping, $T$ is a nonexpansive mapping, and $\left\{\alpha_{n}\right\} \subset[0,1]$. They proved that under some mild conditions, the sequence generated by (1.5) converges in norm to fixed point of nonexpansive mapping, which, in addition, solves the variational inequality (1.2).

Recently, Rizvi et al. [13] suggested and analyzed general viscosity implicit midpoint rule (GVIMR, for short)

$$
x_{n+1}=\alpha_{n} \gamma f\left(x_{n}\right)+\left(1-\alpha_{n} B\right) T\left(\frac{x_{n}+x_{n+1}}{2}\right), n \geqslant 0,
$$

where $\mathrm{f}$ is a contraction mapping, $\mathrm{T}$ is a nonexpansive mapping, and $\mathrm{B}$ is a strongly positive self-adjoint bounded linear operator on $H$ and $\left\{\alpha_{n}\right\} \subset[0,1]$. They proved that the sequence $\left\{x_{n}\right\}$ generated by (1.6) converges strong to the unique solution of VI (1.4).

Our concern now is the following questions.

Question 1.1. Can the algorithms in Moudafi [12], Xu [17], Marino and Xu [11], Alghamdi et al. [1], Xu et al. [19], and Rizvi [13] be extended or improved to more general algorithms? 
Question 1.2. We know that "the Lipschitz operator" is more general than "the contractive operator". What happens if "the contractive operator $f^{\prime \prime}$ in (1.6) is replaced by "the Lipschitz operator U"?

Question 1.3. We know that "the strongly monotone operator and Lipschitz operator" is more general than "the strongly positive bounded linear operator". What will happen if "the strongly positive bounded linear operator B" in (1.6) is replaced by "the strongly monotone operator and Lipschitz operator F"?

The purpose of this article is to give the affirmative answers to these questions mentioned above. Let $\mathrm{F}: \mathrm{H} \rightarrow \mathrm{H}$ be a $\mathrm{K}$-Lipschitzian and $\eta$-strongly monotone mapping, and $\mathrm{U}: \mathrm{H} \rightarrow \mathrm{H}$ be a $\tau$-Lipschitzian mapping. Motivated by the above facts, in this paper, we propose and analyze a more general viscosity implicit midpoint rule (MGVIMR for short)

$$
x_{n+1}=\alpha_{n} \rho U\left(x_{n}\right)+\beta_{n} x_{n}+\left(\left(1-\beta_{n}\right) I-\alpha_{n} \mu F\right) T\left(\frac{x_{n}+x_{n+1}}{2}\right), \forall n \geqslant 0 .
$$

Based on the more general viscosity implicit midpoint iterative scheme, we prove the strong convergence theorem for nonexpansive mapping under certain assumptions, imposed on the sequence of parameters, which, in addition, is the unique solution of variational inequality problem. Furthermore, applications to variational inequalities, hierarchical minimization problems, Fredholm integral equations, and nonlinear evolution equations are discussed. The results and methods presented here extend and generalize the corresponding results and methods given in $[1,11-13,17,19]$.

\section{Preliminaries}

We recall some concepts and results which are needed in sequel.

Definition 2.1. A mapping $\mathrm{T}: \mathrm{H} \rightarrow \mathrm{H}$ is said to be L-Lipschitzian if there exists a constant $\mathrm{L}>0$ such that

$$
\|T x-T y\| \leqslant L\|x-y\|, \quad \forall x, y \in H,
$$

in the case of $L=\alpha \in(0,1)$, $T$ is said to be an $\alpha$-contractive mapping; in the case of $L=1$, $T$ is said to be nonexpansive. We use $\operatorname{Fix}(\mathrm{T})$ to denote the set of fixed points of $\mathrm{T}$.

It is clear that the Lipschitz operator is more general than the contractive operator.

Definition 2.2. A mapping $A: C \rightarrow H$ is called

(i) monotone if

$$
\langle A x-A y, x-y\rangle \geqslant 0, \quad \forall x, y \in C ;
$$

(ii) $\eta$-strongly monotone if there exists a constant $\eta>0$ such that

$$
\langle A x-A y, x-y\rangle \geqslant \eta\|x-y\|^{2}, \quad \forall x, y \in C ;
$$

(iii) $\zeta$-inverse-strongly monotone if there exists a constant $\zeta>0$ such that

$$
\langle A x-A y, x-y\rangle \geqslant \zeta\|A x-A y\|^{2}, \forall x, y \in C .
$$

It is clear that if $A$ is $\zeta$-inverse-strongly monotone, then $A$ is monotone and $\frac{1}{\zeta}$-Lipschitz continuous. Moreover, one also has that, for all $u, v \in C$ and $\lambda>0$,

$$
\|(I-\lambda A) u-(I-\lambda A) v\|^{2} \leqslant\|u-v\|^{2}+\lambda(\lambda-2 \zeta)\|A u-A v\|^{2} .
$$

So, if $\lambda<2 \zeta$, then $I-\lambda A$ is nonexpansive mapping. 
Definition 2.3. An operator $B: H \rightarrow H$ is said to be strongly positive bounded linear operator, if there exists a constant $\bar{\gamma}>0$ such that

$$
\langle\mathrm{B} x, x\rangle \geqslant \bar{\gamma}\|x\|^{2}, \quad \forall x \in \mathrm{H} .
$$

It is clear that if B is strongly positive bounded linear, then B is strongly monotone and Lipschitzian. So, the strongly monotone operator and Lipschitz operator is more general than the strongly positive bounded linear operator.

Definition 2.4. The metric (or nearest point) projection from $H$ onto $C$ is the mapping $P_{C}: H \rightarrow C$ which assigns to each point $x \in H$ the unique point $P_{C} x \in C$ satisfying the property

$$
\left\|x-P_{C} x\right\|=\inf _{y \in C}\|x-y\|:=d(x, C) .
$$

Some important properties of projections are gathered in the following proposition.

Proposition 2.5. For given $\mathrm{x} \in \mathrm{H}$ and $z \in \mathrm{C}$

(i) $z=\mathrm{P}_{\mathrm{C}} \mathrm{x} \Leftrightarrow\langle x-z, y-z\rangle \leqslant 0, \forall y \in \mathrm{C}$;

(ii) $z=\mathrm{P}_{\mathrm{C}} \mathrm{x} \Leftrightarrow\|x-z\|^{2} \leqslant\|x-y\|^{2}-\|y-z\|^{2}, \forall y \in C$;

(iii) $\left\langle P_{C} x-P_{C} y\right\rangle \geqslant\left\|P_{C} x-P_{C} y\right\|^{2}, \forall y \in H$.

Consequently, $\mathrm{P}_{\mathrm{C}}$ is nonexpansive and monotone.

In order to prove our main results, we need the following lemmas.

Lemma 2.6 ([5, 10] (The demiclosedness principle)). Let $\mathrm{H}$ be a Hilbert space, $\mathrm{C}$ a closed convex subset of $\mathrm{H}$, and $\mathrm{T}: \mathrm{C} \rightarrow \mathrm{C}$ a nonexpansive mapping with $\operatorname{Fix}(\mathrm{T}) \neq \emptyset$. If $\left\{\mathrm{x}_{\mathrm{n}}\right\}$ is a sequence in $\mathrm{C}$ such that (i) $\left\{\mathrm{x}_{\mathrm{n}}\right\}$ weakly converges to $\mathrm{x}$ and (ii) $\left\{(\mathrm{I}-\mathrm{T}) \mathrm{x}_{\mathrm{n}}\right\}$ converges strongly to 0 , then $\mathrm{x}=\mathrm{T} \mathrm{x}$.

Lemma $2.7([5,10])$. In real Hilbert space $\mathrm{H}$, the followings hold

(i)

(ii)

$$
\|x+y\|^{2} \leqslant\|x\|^{2}+2\langle y, x+y\rangle, \quad \forall x, y \in H
$$

$$
\|\lambda x+(1-\lambda) y\|^{2}=\lambda\|x\|^{2}+(1-\lambda)\|y\|^{2}-\lambda(1-\lambda)\|x-y\|^{2}
$$

for all $x, y \in \mathrm{H}$ and $\lambda \in(0,1)$.

Lemma 2.8 ([20]). Let $\mathrm{H}$ be a real Hilbert space. Let $\mathrm{V}: \mathrm{H} \rightarrow \mathrm{H}$ be an l-Lipschitzian mapping with a constant $\mathrm{l} \geqslant 0$, and let $\mathrm{G}: \mathrm{H} \rightarrow \mathrm{H}$ be a $\mathrm{k}$-Lipschitzian and $\eta$-strongly monotone mapping with constants $\mathrm{\kappa}, \eta>0$. Then for $0 \leqslant \gamma l<\mu \eta$,

$$
\langle(\mu \mathrm{G}-\gamma \mathrm{V}) x-(\mu \mathrm{G}-\gamma \mathrm{V}) \mathrm{y}, x-\mathrm{y}\rangle \geqslant(\mu \eta-\gamma \mathrm{l})\|x-y\|^{2}, \quad \forall x, y \in \mathrm{C} .
$$

That is, $\mu \mathrm{G}-\gamma \mathrm{V}$ is strongly monotone with constant $\mu \eta-\gamma \mathrm{l}$.

Lemma 2.9 ([17]). Assume $\left\{a_{n}\right\}$ is a sequence of nonnegative real numbers such that

$$
a_{n+1} \leqslant\left(1-\gamma_{n}\right) a_{n}+\delta_{n}, \quad \forall n \geqslant 0,
$$

where $\left\{\gamma_{n}\right\}$ is a sequence in $(0,1)$ and $\left\{\delta_{n}\right\}$ is a sequence in $R$ such that

(i) $\sum_{n=1}^{\infty} \gamma_{n}=\infty$, and

(ii) either $\limsup _{n \rightarrow \infty} \delta_{n} / \gamma_{n} \leqslant 0$ or $\sum_{n=1}^{\infty}\left|\delta_{n}\right|<\infty$.

Then $\lim _{n \rightarrow \infty} a_{n}=0$. 


\section{More general viscosity implicit midpoint rule}

In this section, we prove a strong convergence theorem based on a more general viscosity implicit midpoint rule for fixed point of nonexpansive mapping.

Theorem 3.1. Let $\mathrm{H}$ be a Hilbert space and $\mathrm{T}: \mathrm{H} \rightarrow \mathrm{H}$ a nonexpansive mapping with $\operatorname{Fix}(\mathrm{T}) \neq \emptyset$. Let $\mathrm{F}: \mathrm{H} \rightarrow \mathrm{H}$ be a $\mathrm{k}$-Lipschitzian and $\eta$-strongly monotone mapping with constants $\kappa, \eta \geqslant 0$ and $\mathrm{U}: \mathrm{H} \rightarrow \mathrm{H}$ be a $\tau$-Lipschitzian mapping with constant $\tau>0$. Constants $\mu>0$ and $\rho \geqslant 0$ satisfy $0<\mu<\frac{2 \eta}{\kappa^{2}}$ and $0 \leqslant \rho \tau<\nu$, where $v=1-\sqrt{1-\mu\left(2 \eta-\mu k^{2}\right)}$. Let the iterative sequence $\left\{x_{n}\right\}$ be generated by the following more general viscosity implicit midpoint iterative scheme:

$$
x_{n+1}=\alpha_{n} \rho U\left(x_{n}\right)+\beta_{n} x_{n}+\left(\left(1-\beta_{n}\right) I-\alpha_{n} \mu F\right) T\left(\frac{x_{n}+x_{n+1}}{2}\right), \quad \forall n \geqslant 0,
$$

where $\left\{\alpha_{n}\right\}$ and $\left\{\beta_{n}\right\} \in(0,1)$ for all $n$, and satisfying the following conditions:

(C1) $\lim _{n \rightarrow \infty} \alpha_{n}=0$;

(C2) $\sum_{n=0}^{\infty} \alpha_{n}=\infty$;

(C3) $\sum_{n=0}^{\infty}\left|\alpha_{n+1}-\alpha_{n}\right|<\infty$ and $\sum_{n=0}^{\infty}\left|\beta_{n+1}-\beta_{n}\right|<\infty$.

Then the sequence $\left\{x_{n}\right\}$ converges strongly to $z \in \operatorname{Fix}(T)$, where $z=\mathrm{P}_{\operatorname{Fix}(\mathrm{T})}(\mathrm{I}-\mu \mathrm{F}+\rho \mathrm{U})(z)$. In other words, which is also the unique solution of the variational inequality

$$
\langle(\rho \mathrm{U}-\mu \mathrm{F})(z), x-z\rangle \leqslant 0, \quad \forall x \in \operatorname{Fix}(\mathrm{T}) .
$$

Proof. Since $\mathrm{F}: \mathrm{H} \rightarrow \mathrm{H}$ is $\mathrm{k}$-Lipschitzian continuous and $\eta$-strongly monotone mapping, and $\mathrm{U}: \mathrm{H} \rightarrow \mathrm{H}$ is $\tau$-Lipschitzian continuous mapping, we have

$$
\begin{aligned}
\|(I-\mu F) x-(I-\mu F) y\|^{2} & =\|x-y\|^{2}-2 \mu\langle x-y, F x-F y\rangle+\mu^{2}\|F x-F y\|^{2} \\
& \leqslant\left(1-2 \mu \eta+\mu^{2} \kappa^{2}\right)\|x-y\|^{2} \\
& =(1-v)^{2}\|x-y\|^{2},
\end{aligned}
$$

where $v=1-\sqrt{1-\mu\left(2 \eta-\mu \mathrm{K}^{2}\right)}$, and hence

$$
\begin{aligned}
\left\|P_{\operatorname{Fix}(T)}(I-\mu F+\rho U) x-P_{\operatorname{Fix}(T)}(I-\mu F+\rho U) y\right\| & \leqslant\|(I-\mu F+\rho U) x-(I-\mu F+\rho U) y\| \\
& \leqslant\|(I-\mu F) x-(I-\mu F) y\|+\rho\|U x-U y\| \\
& \leqslant(1-v)\|x-y\|+\rho \tau\|x-y\| \\
& \leqslant(1-(\nu-\rho \tau))\|x-y\|
\end{aligned}
$$

for all $x, y \in H$. Therefore, $P_{\operatorname{Fix}(T)}(I-\mu F+\rho U)$ is a contraction of $H$ into itself, which implies that there exists a unique $z \in \mathrm{H}$ such that

$$
z=\mathrm{P}_{\mathrm{Fix}(\mathrm{T})}(\mathrm{I}-\mu \mathrm{F}+\rho \mathrm{U}) z .
$$

We divide the proof into several steps.

Step 1. We prove that $\left\{x_{n}\right\}$ is bounded. To see this, we take $p \in \operatorname{Fix}(T)$ to deduce that

$$
\begin{aligned}
\left\|x_{n+1}-p\right\|= & \left\|\alpha_{n} \rho U\left(x_{n}\right)+\beta_{n} x_{n}+\left(\left(1-\beta_{n}\right) I-\alpha_{n} \mu F\right) T\left(\frac{x_{n}+x_{n+1}}{2}\right)-p\right\| \\
\leqslant & \left\|\left(\left(1-\beta_{n}\right) I-\alpha_{n} \mu F\right) T\left(\frac{x_{n}+x_{n+1}}{2}\right)-\left(\left(1-\beta_{n}\right) I-\alpha_{n} \mu F\right) T(p)\right\| \\
& +\left\|\alpha_{n}\left(\rho U\left(x_{n}\right)-\mu F(p)\right)\right\|+\left\|\beta_{n}\left(x_{n}-p\right)\right\|
\end{aligned}
$$




$$
\begin{aligned}
\leqslant & \left(1-\beta_{n}\right)\left\|\left(I-\frac{\alpha_{n} \mu}{1-\beta_{n}} F\right) T\left(\frac{x_{n}+x_{n+1}}{2}\right)-\left(I-\frac{\alpha_{n} \mu}{1-\beta_{n}} F\right) T(p)\right\| \\
& +\alpha_{n}\left\|\rho U\left(x_{n}\right)-\mu F(p)\right\|+\beta_{n}\left\|x_{n}-p\right\| \\
\leqslant & \left(1-\beta_{n}\right)\left\|\left(I-\frac{\alpha_{n} \mu}{1-\beta_{n}} F\right) T\left(\frac{x_{n}+x_{n+1}}{2}\right)-\left(I-\frac{\alpha_{n} \mu}{1-\beta_{n}} F\right) T(p)\right\| \\
& +\alpha_{n}\left\|\rho U\left(x_{n}\right)-\rho U(p)+\rho U(p)-\mu F(p)\right\|+\beta_{n}\left\|x_{n}-p\right\| \\
\leqslant & \left(1-\beta_{n}\right)\left(1-\frac{\alpha_{n} v}{1-\beta_{n}}\right)\left\|T\left(\frac{x_{n}+x_{n+1}}{2}\right)-T(p)\right\| \\
& +\alpha_{n} \rho \tau\left\|x_{n}-p\right\|+\alpha_{n}\|(\rho U-\mu F) p\|+\beta_{n}\left\|x_{n}-p\right\| \\
\leqslant & \frac{1-\beta_{n}-\alpha_{n} v}{2}\left(\left\|x_{n}-p\right\|+\left\|x_{n+1}-p\right\|\right) \\
& +\alpha_{n} \rho \tau\left\|x_{n}-p\right\|+\alpha_{n}\|(\rho U-\mu F) p\|+\beta_{n}\left\|x_{n}-p\right\| \\
= & \frac{1-\beta_{n}-\alpha_{n} v}{2}\left\|x_{n+1}-p\right\|+\frac{1+\beta_{n}+(2 \rho \tau-v) \alpha_{n}}{2}\left\|x_{n}-p\right\|+\alpha_{n}\|(\rho U-\mu F) p\| .
\end{aligned}
$$

It follows that

$$
\frac{1+\beta_{n}+\alpha_{n} v}{2}\left\|x_{n+1}-p\right\| \leqslant \frac{1+\beta_{n}+(2 \rho \tau-v) \alpha_{n}}{2}\left\|x_{n}-p\right\|+\alpha_{n}\|(\rho U-\mu F) p\|,
$$

and, moreover,

$$
\begin{aligned}
\left\|x_{n+1}-p\right\| & \leqslant \frac{1+\beta_{n}+(2 \rho \tau-v) \alpha_{n}}{1+\beta_{n}+\alpha_{n} v}\left\|x_{n}-p\right\|+\frac{2 \alpha_{n}}{1+\beta_{n}+\alpha_{n} v}\|(\rho U-\mu F) p\| \\
& =\left(1-\frac{2 \alpha_{n}(v-\rho \tau)}{1+\beta_{n}+\alpha_{n} v}\right)\left\|x_{n}-p\right\|+\frac{2 \alpha_{n}(v-\rho \tau)}{1+\beta_{n}+\alpha_{n} v}\left(\frac{1}{v-\rho \tau}\|(\rho U-\mu F) p\|\right) \\
& \left.\leqslant \max _{\{}\left\|x_{n}-p\right\|, \frac{1}{v-\rho \tau}\|(\rho U-\mu F) p\|\right\} .
\end{aligned}
$$

By induction, we readily obtain

$$
\left\|x_{n+1}-p\right\| \leqslant \max \left\{\left\|x_{0}-p\right\|, \frac{1}{v-\rho \tau}\|(\rho U-\mu F) p\|\right\}
$$

for all $n$ and $x_{0} \in C$. It turns out that $\left\{x_{n}\right\}$ is bounded.

Step 2. $\lim _{n \rightarrow \infty}\left\|x_{n+1}-x_{n}\right\|=0$. To see this, we apply (3.1) to get

$$
\begin{aligned}
\left\|x_{n+1}-x_{n}\right\|= & \| \alpha_{n} \rho U\left(x_{n}\right)+\beta_{n} x_{n}+\left(\left(1-\beta_{n}\right) I-\alpha_{n} \mu F\right) T\left(\frac{x_{n}+x_{n+1}}{2}\right) \\
& -\left[\alpha_{n-1} \rho U\left(x_{n-1}\right)+\beta_{n-1} x_{n-1}+\left(\left(1-\beta_{n-1}\right) I-\alpha_{n-1} \mu \mathrm{F}\right) \mathrm{T}\left(\frac{x_{n-1}+x_{n}}{2}\right)\right] \| \\
\leqslant & \| \alpha_{n} \rho\left(U\left(x_{n}\right)-U\left(x_{n-1}\right)\right)+\left(\alpha_{n}-\alpha_{n-1}\right) \rho U\left(x_{n-1}\right)+\beta_{n}\left(x_{n}-x_{n-1}\right)+\left(\beta_{n}-\beta_{n-1}\right) x_{n-1} \\
& +\left(1-\beta_{n}\right)\left[\left(I-\frac{\alpha_{n} \mu}{1-\beta_{n}} \mathrm{~F}\right) \mathrm{T}\left(\frac{x_{n}+x_{n+1}}{2}\right)-\left(I-\frac{\alpha_{n} \mu}{1-\beta_{n}} \mathrm{~F}\right) \mathrm{T}\left(\frac{x_{n-1}+x_{n}}{2}\right)\right] \\
& +\left[\left(\left(1-\beta_{n}\right) \mathrm{I}-\alpha_{n} \mu \mathrm{F}\right)-\left(\left(1-\beta_{n-1}\right) \mathrm{I}-\alpha_{n-1} \mu \mathrm{F}\right)\right] \mathrm{T}\left(\frac{x_{n-1}+x_{n}}{2}\right) \| \\
\leqslant & \alpha_{n} \rho \tau\left\|x_{n}-x_{n-1}\right\|+\beta_{n}\left\|x_{n}-x_{n-1}\right\|+\left(1-\beta_{n}\right)\left(1-\frac{\alpha_{n} v}{1-\beta_{n}}\right)\left\|\mathrm{T}\left(\frac{x_{n}+x_{n+1}}{2}\right)-\mathrm{T}\left(\frac{x_{n-1}+x_{n}}{2}\right)\right\| \\
& +\left|\alpha_{n}-\alpha_{n-1}\right|\left(\left\|\rho U\left(x_{n-1}\right)\right\|+\left\|\mu F T\left(\frac{x_{n-1}+x_{n}}{2}\right)\right\|\right) \\
& +\left|\beta_{n}-\beta_{n-1}\right|\left(\left\|x_{n-1}\right\|+\left\|T\left(\frac{x_{n-1}+x_{n}}{2}\right)\right\|\right)
\end{aligned}
$$




$$
\begin{aligned}
\leqslant & \alpha_{n} \rho \tau\left\|x_{n}-x_{n-1}\right\|+\beta_{n}\left\|x_{n}-x_{n-1}\right\|+\frac{1-\beta_{n}-\alpha_{n} v}{2}\left(\left\|x_{n+1}-x_{n}\right\|+\left\|x_{n}-x_{n-1}\right\|\right) \\
& +M\left(\left|\alpha_{n}-\alpha_{n-1}\right|+\left|\beta_{n}-\beta_{n-1}\right|\right) \\
= & \frac{1-\beta_{n}-\alpha_{n} v}{2}\left\|x_{n+1}-x_{n}\right\|+\frac{1+\beta_{n}+(2 \rho \tau-v) \alpha_{n}}{2}\left\|x_{n}-x_{n-1}\right\| \\
& +M\left(\left|\alpha_{n}-\alpha_{n-1}\right|+\left|\beta_{n}-\beta_{n-1}\right|\right) .
\end{aligned}
$$

Here $M>0$ is a constant such that

$$
M=\max \left\{\sup _{n \geqslant 0}\left(\left\|x_{n}\right\|+T\left(\frac{x_{n}+x_{n+1}}{2}\right)\right), \sup _{n \geqslant 0}\left(\left\|\rho U\left(x_{n}\right)\right\|+\left\|\mu \mathrm{FT}\left(\frac{x_{n}+x_{n+1}}{2}\right)\right\|\right)\right\} .
$$

It turns out that

$$
\frac{1+\beta_{n}+\alpha_{n} v}{2}\left\|x_{n+1}-x_{n}\right\| \leqslant \frac{1+\beta_{n}+(2 \rho \tau-v) \alpha_{n}}{2}\left\|x_{n}-x_{n-1}\right\|+M\left(\left|\alpha_{n}-\alpha_{n-1}\right|+\left|\beta_{n}-\beta_{n-1}\right|\right) .
$$

Consequently, we arrive at

$$
\begin{aligned}
\left\|x_{n+1}-x_{n}\right\| & \leqslant \frac{1+\beta_{n}+(2 \rho \tau-v) \alpha_{n}}{1+\beta_{n}+\alpha_{n} v}\left\|x_{n}-x_{n-1}\right\|+\frac{2 M}{1+\beta_{n}+\alpha_{n} \nu}\left(\left|\alpha_{n}-\alpha_{n-1}\right|+\left|\beta_{n}-\beta_{n-1}\right|\right) \\
& =\left(1-\frac{2 \alpha_{n}(v-\rho \tau)}{1+\beta_{n}+\alpha_{n} v}\right)\left\|x_{n}-x_{n-1}\right\|+\frac{2 M}{1+\beta_{n}+\alpha_{n} v}\left(\left|\alpha_{n}-\alpha_{n-1}\right|+\left|\beta_{n}-\beta_{n-1}\right|\right) .
\end{aligned}
$$

By using the conditions (C1)-(C3), we can apply Lemma 2.9 to (3.3) to obtain $\left\|x_{n+1}-x_{n}\right\| \rightarrow 0$ as $n \rightarrow \infty$, as required.

Step 3. $\lim _{n \rightarrow \infty}\left\|x_{n}-T x_{n}\right\|=0$. This follows from the argument below:

$$
\begin{aligned}
\left\|x_{n}-T x_{n}\right\| \leqslant & \left\|x_{n}-x_{n+1}\right\|+\left\|x_{n+1}-T\left(\frac{x_{n}+x_{n+1}}{2}\right)\right\|+\left\|T\left(\frac{x_{n}+x_{n+1}}{2}\right)-T\left(x_{n}\right)\right\| \\
\leqslant & \left\|x_{n}-x_{n+1}\right\|+\alpha_{n}\left\|\rho U\left(x_{n}\right)-\mu F T\left(\frac{x_{n}+x_{n+1}}{2}\right)\right\|+\beta_{n}\left\|x_{n}-T\left(\frac{x_{n}+x_{n+1}}{2}\right)\right\|+\frac{1}{2}\left\|x_{n+1}-x_{n}\right\| \\
\leqslant & \frac{3}{2}\left\|x_{n}-x_{n+1}\right\|+\alpha_{n}\left\|\rho U\left(x_{n}\right)-\mu F T\left(\frac{x_{n}+x_{n+1}}{2}\right)\right\| \\
& +\beta_{n}\left(\left\|x_{n}-T x_{n}\right\|+\left\|T x_{n}-T\left(\frac{x_{n}+x_{n+1}}{2}\right)\right\|\right) \\
= & \frac{1}{2}\left(3+\beta_{n}\right)\left\|x_{n}-x_{n+1}\right\|+\alpha_{n}\left\|\rho U\left(x_{n}\right)-\mu F T\left(\frac{x_{n}+x_{n+1}}{2}\right)\right\|+\beta_{n}\left\|x_{n}-T x_{n}\right\|,
\end{aligned}
$$

and therefore

$$
\left\|x_{n}-T x_{n}\right\| \leqslant \frac{3+\beta_{n}}{2\left(1-\beta_{n}\right)}\left\|x_{n}-x_{n+1}\right\|+\frac{\alpha_{n}}{1-\beta_{n}}\left\|\rho U\left(x_{n}\right)-\mu F T\left(\frac{x_{n}+x_{n+1}}{2}\right)\right\| .
$$

Since $\lim _{n \rightarrow \infty}\left\|x_{n}-x_{n+1}\right\|=0$ and $\alpha_{n} \rightarrow 0$ as $n \rightarrow \infty$, we obtain

$$
\lim _{n \rightarrow \infty}\left\|x_{n}-T x_{n}\right\|=0 .
$$

Step 4. We prove that $\omega_{w}\left(x_{n}\right) \subset \operatorname{Fix}(T)$. Here

$$
\omega_{w}\left(x_{n}\right)=\left\{x \in H \text { : there exists a subsequence of }\left\{x_{n}\right\} \text { weakly converging to } x\right\}
$$

is the weak $\omega$-limit set of $\left\{x_{n}\right\}$. This is now a straightforward consequence of Step 3 and Lemma 2.6. 
Step 5. We claim that

$$
\limsup _{n \rightarrow \infty}\left\langle(\rho U-\mu F) z, x_{n}-z\right\rangle \leqslant 0,
$$

where $z \in \operatorname{Fix}(T)$ is the unique solution of variational inequality (3.2).

As a matter of fact, we can find a subsequence $\left\{x_{n_{i}}\right\}$ of $\left\{x_{n}\right\}$ such that $\left\{x_{n_{i}}\right\}$ converges weakly to a point $\omega$ and moreover

$$
\limsup _{n \rightarrow \infty}\left\langle(\rho U-\mu F) z, x_{n}-z\right\rangle=\lim _{i \rightarrow \infty}\left\langle(\rho U-\mu F) z, x_{n_{i}}-z\right\rangle .
$$

Since $\omega \in \operatorname{Fix}(T)$ by Step 4, we can combine (3.4) and (3.5) and use Proposition 2.5 (i) to conclude

$$
\limsup _{n \rightarrow \infty}\left\langle(\rho U-\mu F) z, x_{n}-z\right\rangle=\lim _{i \rightarrow \infty}\left\langle(\rho U-\mu F) z, x_{n_{i}}-z\right\rangle=\langle(\rho U-\mu F) z, \omega-z\rangle \leqslant 0 .
$$

Step 6. We finally prove that $x_{n} \rightarrow z$ in norm. Here agian $z \in \operatorname{Fix}(T)$ is the unique solution of variational inequality (3.2). We present the details as follows:

$$
\begin{aligned}
\left\|x_{n+1}-z\right\|^{2}= & \left\langle\alpha_{n} \rho U\left(x_{n}\right)+\beta_{n} x_{n}+\left(\left(1-\beta_{n}\right) I-\alpha_{n} \mu F\right) T\left(\frac{x_{n}+x_{n+1}}{2}\right)-z, x_{n+1}-z\right\rangle \\
= & \left\langle\alpha_{n}\left(\rho U\left(x_{n}\right)-\mu F(z)\right)+\beta_{n}\left(x_{n}-z\right)\right. \\
& \left.+\left(1-\beta_{n}\right)\left[\left(I-\frac{\alpha_{n} \mu}{1-\beta_{n}} F\right) T\left(\frac{x_{n}+x_{n+1}}{2}\right)-\left(I-\frac{\alpha_{n} \mu}{1-\beta_{n}} F\right) T(z)\right], x_{n+1}-z\right\rangle \\
\leqslant & \alpha_{n} \rho\left\langle\left(U\left(x_{n}\right)-U(z)\right), x_{n+1}-z\right\rangle+\alpha_{n}\left\langle(\rho U-\mu F)(z), x_{n+1}-z\right\rangle+\beta_{n}\left\langle x_{n}-z, x_{n+1}-z\right\rangle \\
& +\left(1-\beta_{n}\right)\left\langle\left[\left(I-\frac{\alpha_{n} \mu}{1-\beta_{n}} F\right) T\left(\frac{x_{n}+x_{n+1}}{2}\right)-\left(I-\frac{\alpha_{n} \mu}{1-\beta_{n}} F\right) T(z)\right], x_{n+1}-z\right\rangle \\
\leqslant & \left(\alpha_{n} \rho \tau+\beta_{n}\right)\left\|x_{n}-z\right\|\left\|x_{n+1}-z\right\|+\alpha_{n}\left\langle(\rho U-\mu F)(z), x_{n+1}-z\right\rangle \\
& +\left(1-\beta_{n}-\alpha_{n} v\right)\left\|T\left(\frac{x_{n}+x_{n+1}}{2}\right)-T(z)\right\|\left\|x_{n+1}-z\right\| \\
\leqslant & \left(\alpha_{n} \rho \tau+\beta_{n}\right)\left\|x_{n}-z\right\|\left\|x_{n+1}-z\right\|+\alpha_{n}\left\langle(\rho U-\mu F)(z), x_{n+1}-z\right\rangle \\
& +\frac{1-\beta_{n}-\alpha_{n} v}{2}\left(\left\|x_{n}-z\right\|+\left\|x_{n+1}-z\right\|\right)\left\|x_{n+1}-z\right\| \\
= & \frac{1-\beta_{n}-\alpha_{n} v}{2}\left\|x_{n+1}-z\right\|^{2}+\frac{1+\beta_{n}+(2 \rho \tau-v) \alpha_{n}}{2}\left\|x_{n}-z\right\|\left\|x_{n+1}-z\right\| \\
& +\alpha_{n}\left\langle(\rho U-\mu F)(z), x_{n+1}-z\right\rangle .
\end{aligned}
$$

It turns out that

$$
\frac{1+\beta_{n}+\alpha_{n} v}{2}\left\|x_{n+1}-z\right\|^{2} \leqslant \frac{1+\beta_{n}+(2 \rho \tau-v) \alpha_{n}}{2}\left\|x_{n}-z\right\|\left\|x_{n+1}-z\right\|+\alpha_{n}\left\langle(\rho U-\mu F)(z), x_{n+1}-z\right\rangle .
$$

Consequently, we arrive at

$$
\begin{aligned}
\left\|x_{n+1}-z\right\|^{2} & \leqslant \frac{1+\beta_{n}+(2 \rho \tau-v) \alpha_{n}}{1+\beta_{n}+\alpha_{n} v}\left\|x_{n}-z\right\|\left\|x_{n+1}-z\right\|+\frac{2 \alpha_{n}}{1+\beta_{n}+\alpha_{n} v}\left\langle(\rho U-\mu F)(z), x_{n+1}-z\right\rangle \\
& \leqslant \frac{1+\beta_{n}+(2 \rho \tau-v) \alpha_{n}}{2\left(1+\beta_{n}+\alpha_{n} v\right)}\left(\left\|x_{n}-z\right\|^{2}+\left\|x_{n+1}-z\right\|^{2}\right)+\frac{2 \alpha_{n}}{1+\beta_{n}+\alpha_{n} v}\left\langle(\rho U-\mu F)(z), x_{n+1}-z\right\rangle .
\end{aligned}
$$

Hence, we get

$$
\frac{1+\beta_{n}+(3 v-2 \rho \tau)}{2\left(1+\beta_{n}+\alpha_{n} v\right) \alpha_{n}}\left\|x_{n+1}-z\right\|^{2} \leqslant \frac{1+\beta_{n}+(2 \rho \tau-v) \alpha_{n}}{2\left(1+\beta_{n}+\alpha_{n} v\right)}\left\|x_{n}-z\right\|^{2} \frac{2 \alpha_{n}}{1+\beta_{n}+\alpha_{n} v}\left\langle(\rho U-\mu F)(z), x_{n+1}-z\right\rangle,
$$


which implies that

$$
\begin{aligned}
\left\|x_{n+1}-z\right\|^{2} \leqslant & \frac{1+\beta_{n}+(2 \rho \tau-v) \alpha_{n}}{1+\beta_{n}+(3 v-2 \rho \tau) \alpha_{n}}\left\|x_{n}-z\right\|^{2}+\frac{4 \alpha_{n}}{1+\beta_{n}+(3 v-2 \rho \tau) \alpha_{n}} \\
& \times\left\langle(\rho U-\mu F)(z), x_{n+1}-z\right\rangle \\
= & \left(1-\frac{4(v-\rho \tau) \alpha_{n}}{1+\beta_{n}+(3 v-2 \rho \tau) \alpha_{n}}\right)\left\|x_{n}-z\right\|^{2}+\frac{4 \alpha_{n}(v-\rho \tau)}{1+\beta_{n}+(3 v-2 \rho \tau) \alpha_{n}} \\
& \times\left\{\frac{1}{v-\rho \tau}\left\langle(\rho U-\mu F)(z), x_{n+1}-z\right\rangle\right\} \\
= & \left(1-\gamma_{n}\right)\left\|x_{n}-z\right\|^{2}+\gamma_{n} \delta_{n},
\end{aligned}
$$

where $\gamma_{n}=\frac{4(v-\rho \tau) \alpha_{n}}{1+\beta_{n}+(3 v-2 \rho \tau) \alpha_{n}}$ and $\delta_{n}=\frac{1}{v-\rho \tau}\left\langle(\rho U-\mu F)(z), x_{n+1}-z\right\rangle$. Since $\lim _{n \rightarrow \infty} \alpha_{n}=0$ and $\sum_{n=0}^{\infty} \alpha_{n}=\infty$, it is easy to see that $\lim _{n \rightarrow \infty} \gamma_{n}=0, \sum_{n=0}^{\infty} \gamma_{n}=\infty$, and $\limsup _{n \rightarrow \infty} \delta_{n} \leqslant 0$. It follows from (3.6), (3.7), and Lemma 2.9 that $x_{n} \rightarrow z$. This completes the proof.

As a direct consequence of Theorem 3.1, we obtain the following result due to Rizvi [13] for fixed point of nonexpansive mapping. Take $\rho:=\gamma, \mathrm{U}:=\mathrm{f}$ a contraction, $\mu:=1, \beta_{\mathrm{n}}:=0 \mathrm{~F}:=\mathrm{B}$ a strongly positive linear bounded operator in Theorem 3.1, then the following corollary is obtained.

Corollary 3.2. Let $\mathrm{H}$ be a real Hilbert space and $\mathrm{B}: \mathrm{H} \rightarrow \mathrm{H}$ be a strongly positive bounded linear operator with constant $\bar{\gamma}>0$ such that $0<\gamma<\frac{\bar{\gamma}}{\alpha}<\gamma+\frac{1}{\alpha}$ and $\mathrm{f}: \mathrm{H} \rightarrow \mathrm{H}$ be a contraction mapping with constant $\alpha \in(0,1)$. Let $\mathrm{T}: \mathrm{H} \rightarrow \mathrm{H}$ be a nonexpansive mapping such that $\operatorname{Fix}(\mathrm{T}) \neq \emptyset$. Let the iterative sequence $\left\{x_{\mathrm{n}}\right\}$ be generated by the following general viscosity implicit midpoint iterative scheme:

$$
x_{n+1}=\alpha_{n} \gamma f\left(x_{n}\right)+\left(1-\alpha_{n} B\right) T\left(\frac{x_{n}+x_{n+1}}{2}\right), \forall n \geqslant 0,
$$

where $\left\{\alpha_{n}\right\}$ is the sequence in $(0,1)$ and satisfying the following conditions:

(i) $\lim _{n \rightarrow \infty} \alpha_{n}=0$;

(ii) $\sum_{n=0}^{\infty} \alpha_{n}=\infty$;

(iii) $\sum_{n=1}^{\infty}\left|\alpha_{n}-\alpha_{n-1}\right|<\infty$ or $\lim _{n \rightarrow \infty} \frac{\alpha_{n+1}}{\alpha_{n}}=1$.

Then the sequence $\left\{x_{n}\right\}$ converges strongly to $z \in \operatorname{Fix}(T)$, where $z=P_{\operatorname{Fix}(T)}(I-B+\gamma f)(z)$. In other words, which is also the unique solution of variational inequality (1.4).

The following corollary is due to $\mathrm{Xu}$ et al. [19] for fixed point of nonexpansive mapping. Take $\gamma:=1$, $\mathrm{B}:=\mathrm{I}$ in Corollary 3.2, then the following corollary is obtained.

Corollary 3.3. Let $\mathrm{H}$ be a real Hilbert space and $\mathrm{f}: \mathrm{H} \rightarrow \mathrm{H}$ be a contraction mapping with constant $\alpha \in(0,1)$. Let $\mathrm{T}: \mathrm{H} \rightarrow \mathrm{H}$ be a nonexpansive mapping such that $\operatorname{Fix}(\mathrm{T}) \neq \emptyset$. Let the iterative sequence $\left\{x_{\mathrm{n}}\right\}$ generated by the following viscosity implicit midpoint iterative scheme:

$$
x_{n+1}=\alpha_{n} f\left(x_{n}\right)+\left(1-\alpha_{n}\right) T\left(\frac{x_{n}+x_{n+1}}{2}\right), \forall n \geqslant 0,
$$

where $\left\{\alpha_{n}\right\}$ is the sequence in $(0,1)$ and satisfying the conditions (i)-(iii) of Corollary 3.2. Then the sequence $\left\{x_{n}\right\}$ converges strongly to $z \in \operatorname{Fix}(T)$, where $z=\mathrm{P}_{\operatorname{Fix}(\mathrm{T})} \mathrm{f}(z)$, which, in addition, also solves variational inequality (1.2).

The following corollary is due to Alghamdi et al. [1] for fixed point problem of nonexpansive problem of nonexpansive mapping. Take $f:=\mathrm{I}$, in Corollary 3.3, then the following corollary is obtained.

Corollary 3.4. Let $\mathrm{H}$ be a real Hilbert space and $\mathrm{T}: \mathrm{H} \rightarrow \mathrm{H}$ be a nonexpansive mapping such that $\operatorname{Fix}(\mathrm{T}) \neq \emptyset$. Let the iterative sequence $\left\{x_{n}\right\}$ generated by the following implicit midpoint iterative scheme:

$$
x_{n+1}=\alpha_{n} x_{n}+\left(1-\alpha_{n}\right) \mathrm{T}\left(\frac{x_{n}+x_{n+1}}{2}\right), \forall n \geqslant 0,
$$

where $\left\{\alpha_{n}\right\}$ is the sequence in $(0,1)$ and satisfying the conditions (i)-(iii) of Corollary 3.2. Then the sequence $\left\{x_{n}\right\}$ 
converges strongly to $z \in \operatorname{Fix}(T)$.

Remark 3.5. Theorem 3.1 extends and generalizes the general viscosity implicit midpoint rule of Rizvi [13], viscosity implicit midpoint rule of $\mathrm{Xu}$ et al. [19], and implicit midpoint rule of Alghamdi et al. [1] to a more general viscosity implicit midpoint rule for a nonexpansive mappings, which also includes the results of $[11,12,17]$ as special cases.

\section{Applications}

\subsection{Application to variational inequalities}

Consider the variational inequality (VI)

$$
\left\langle A x^{*}, x-x^{*}\right\rangle \geqslant 0, \quad x \in C,
$$

where $A$ is a (single-valued) monotone operator in $H$ and $C$ is a closed convex subset of $H$. We assume $C \subset \operatorname{dom}(A)$. An example of (4.1) is constrained minimization problem

$$
\min _{x \in C} \varphi(x)
$$

where $\varphi: H \rightarrow R$ is a lower-semicontinuous convex function. If $\varphi$ is (Fréchet) differentiable, then the minimization (4.2) is equalivalent reformulated as (4.1) with $A=\nabla \varphi$.

Notice that the VI (4.1) is equivalent to the fixed point problem, for any $\lambda>0$,

$$
\mathrm{T}^{*}=x^{*}, \quad \mathrm{~T} x:=\mathrm{P}_{\mathrm{C}}(\mathrm{I}-\lambda \mathrm{A}) x .
$$

If $A$ is Lipschitzian and strongly monotone, then, for $\lambda>0$ small enough, $T$ is contraction and its unique fixed point is also the unique solution of the VI (4.1). However, if $A$ is not strongly monotone, $T$ is no longer a contraction, in general. In this case we must deal with nonexpansive mappings for solving the VI (4.1). More precisely, we assume

(A1) $A$ is L-Lipschitzian for some $L>0$, that is,

$$
\|A x-A y\| \leqslant L\|x-y\|, \quad x, y \in H
$$

(A2) $A$ is $\mu$-inverse strongly monotone ( $\mu$-ism) for some $\mu>0$, namely

$$
\langle A x-A y, x-y\rangle \geqslant \mu\|A x-A y\|^{2}, x, y \in H .
$$

Note that if $\nabla \varphi$ is L-Lipschitzian, then $\nabla \varphi$ is $\frac{1}{\mathrm{~L}}$-ism.

Under the conditions (A1) and (A2), it is well-known [18] that the operator $T=P_{C}(I-\lambda A)$ is nonexpansive provided $0<\lambda<2 \mu$. It turns out that for this range of value of $\lambda$, fixed point algorithms can be applied to solve VI (4.1). Applying Theorem 3.1 we get the result below.

Theorem 4.1. Assume the VI (4.1) is solvable. Assume also A satisfies (A1) and (A2), and $0<\lambda<2 \mu$. Let $\mathrm{F}: \mathrm{H} \rightarrow \mathrm{H}$ be a $\mathrm{k}$-Lipschitzian and $\eta$-strongly monotone mapping with constants $\mathrm{k}, \eta \geqslant 0$ and $\mathrm{U}: \mathrm{H} \rightarrow \mathrm{H}$ be $a$ $\tau$-Lipschitzian mapping with constant $\tau>0$. Constants $\mu>0$ and $\rho \geqslant 0$ satisfy $0<\mu<\frac{2 \eta}{\kappa^{2}}$ and $0 \leqslant \rho \tau<\nu$, where $v=1-\sqrt{1-\mu\left(2 \eta-\mu \kappa^{2}\right)}$. Let $\left\{x_{n}\right\}$ be generated by the more general viscosity implicit midpoint rule:

$$
x_{n+1}=\alpha_{n} \rho U\left(x_{n}\right)+\beta_{n} x_{n}+\left(\left(1-\beta_{n}\right) I-\alpha_{n} \mu F\right) P_{C}(I-\lambda A)\left(\frac{x_{n}+x_{n+1}}{2}\right), \forall n \geqslant 0 .
$$

In addition, assume the parameters $\left\{\alpha_{n}\right\},\left\{\beta_{n}\right\}$ satisfy the conditions (C1)-(C3) of Theorem 3.1. Then the sequence $\left\{x_{n}\right\}$ converges in norm to a solution $x^{*}$ of the VI (4.1) which is also a solution to the VI

$$
\left\langle(\rho \mathrm{U}-\mu \mathrm{F}) x^{*}, x-x^{*}\right\rangle \leqslant 0, \quad \forall x \in A^{-1}(0) .
$$




\subsection{Application to hierarchical minimization}

We next consider a hierarchical minimization problem (see [7] and references therein).

Let $\varphi_{0}, \varphi_{1}: \mathrm{H} \rightarrow \mathrm{R}$ be lower semicontinuous convex functions. Consider the hierarchical minimization

$$
\min _{x \in S_{0}} \varphi_{1}(x), \quad S_{0}=\arg \min _{x \in \mathcal{H}} \varphi_{0}(x) .
$$

Here we always assume that $S_{0}$ is nonempty. Let $S=\arg \min _{x \in S_{0}} \varphi_{1}(x)$ and $S \neq \emptyset$.

Assume $\varphi_{0}$ and $\varphi_{1}$ are differentiable and their gradients satisfy the Lipschitz continuity conditions:

$$
\left\|\nabla \varphi_{0}(x)-\nabla \varphi_{0}(\mathrm{y})\right\| \leqslant \mathrm{L}_{0}\|x-\mathrm{y}\|, \quad\left\|\nabla \varphi_{1}(\mathrm{x})-\nabla \varphi_{1}(\mathrm{y})\right\| \leqslant \mathrm{L}_{1}\|x-\mathrm{y}\| .
$$

Note that the condition (4.4) implies that $\nabla \varphi_{i}$ is $\frac{1}{\mathrm{~L}_{i}}$-ism $(i=0,1)$. Now let

$$
\mathrm{T}_{0}=\mathrm{I}-\gamma_{0} \nabla \varphi_{0}, \quad \mathrm{~T}_{1}=\mathrm{I}-\gamma_{1} \nabla \varphi_{1},
$$

where $\gamma_{0}>0$ and $\gamma_{1}>0$. Note that $T_{i}$ is (averaged) nonexpansive [18] if $0<\gamma_{i}<2 / L_{i}(i=0,1)$. Also, it is easily seen that $S_{0}=\operatorname{Fix}\left(T_{0}\right)$.

The optimality condition for $x^{*} \in S_{0}$ to be a solution of the hierarchical minimization (4.3) is the VI

$$
x^{*} \in S_{0}, \quad\left\langle\nabla \varphi_{1}\left(x^{*}\right), x-x^{*}\right\rangle \geqslant 0, \quad x \in S_{0} .
$$

This is the VI (4.1) with $C=S_{0}$ and $A=\nabla \varphi_{1}$. Therefore, we have the following result.

Theorem 4.2. Assume the hierarchical minimization problem (4.3) is solvable. Assume (4.4) and $0<\gamma_{i}<$ $2 / \mathrm{L}_{i},(i=0,1)$. Let $\mathrm{F}: \mathrm{H} \rightarrow \mathrm{H}$ be a $\mathrm{k}$-Lipschitzian and $\eta$-strongly monotone mapping with constants $\mathrm{k}, \eta \geqslant 0$ and $\mathrm{U}: \mathrm{H} \rightarrow \mathrm{H}$ be a $\tau$-Lipschitzian mapping with constant $\tau>0$. Constants $\mu>0$ and $\rho \geqslant 0$ satisfy $0<\mu<\frac{2 \eta}{\kappa^{2}}$ and $0 \leqslant \rho \tau<v$, where $v=1-\sqrt{1-\mu\left(2 \eta-\mu \kappa^{2}\right)}$. Let $\left\{x_{n}\right\}$ be generated by the more general viscosity implicit midpoint rule:

$$
x_{n+1}=\alpha_{n} \rho U\left(x_{n}\right)+\beta_{n} x_{n}+\left(\left(1-\beta_{n}\right) I-\alpha_{n} \mu F\right) P_{S_{0}}\left(I-\lambda \nabla \varphi_{1}\right)\left(\frac{x_{n}+x_{n+1}}{2}\right), \quad \forall n \geqslant 0 .
$$

In addition, assume the parameters $\left\{\alpha_{n}\right\},\left\{\beta_{n}\right\}$ satisfy the conditions (C1)-(C3) of Theorem 3.1. Then the sequence $\left\{x_{n}\right\}$ converges in norm to a solution $x^{*}$ of the VI (4.1) which is also a solution to the VI

$$
\left\langle(\rho \mathrm{U}-\mu \mathrm{F}) x^{*}, x-x^{*}\right\rangle \leqslant 0, \quad \forall x \in S .
$$

\subsection{Application to Fredholm integral equation}

Consider a Fredholm integral equation of the following form

$$
x(t)=g(t)+\int_{0}^{t} F(t, s, x(s)) d s, t \in[0,1],
$$

where $g$ is a continuous function on $[0,1]$ and $F:[0,1] \times[0,1] \times R \rightarrow R$ is continuous. Note that if $F$ satisfies the Lipschitz continuity condition, i.e.,

$$
|F(t, s, x)-F(t, s, y)| \leqslant|x-y|, \quad \forall t, s \in[0,1], \quad x, y \in R,
$$

then, equation (4.5) has at least one solution in $\mathrm{L}^{2}[0,1]$ (see [10]). Define a mapping $S: \mathrm{L}^{2}[0,1] \rightarrow \mathrm{L}^{2}[0,1]$ by

$$
(S x)(t)=g(t)+\int_{0}^{t} F(s, t, x(s)) d s, \quad \forall t \in[0,1]
$$


It is easy to see that $S$ is nonexpansive. In fact, we have for $x, y \in \mathrm{L}^{2}[0,1]$,

$$
\begin{aligned}
\|S x-S y\|^{2} \leqslant \int_{0}^{1}|(S x)(t)-(S y)(t)|^{2} d t & =\left.\int_{0}^{1}|| \int_{0}^{1}(F(t, s, x(s))-F(t, s, y(s))) d s\right|^{2} d t \\
& \leqslant \int_{0}^{1}\left|\int_{0}^{1}\right| x(s)-y(s)|d s|^{2} d t=\int_{0}^{1}|x(s)-y(s)|^{2} d s=\|x-y\|^{2} .
\end{aligned}
$$

This means that finding the solution of integral equation (4.5) is reduced to find a fixed point of the nonexpansive mapping $S$ in the Hilbert space $\mathrm{L}^{2}[0,1]$. Initiating with any function $x_{0} \in \mathrm{L}^{2}[0,1]$. The sequence of functions $\left\{x_{n}\right\}$ in $L^{2}[0,1]$ generated by the more general viscosity implicit midpoint iterative scheme:

$$
x_{n+1}=\alpha_{n} \rho U\left(x_{n}\right)+\beta_{n} x_{n}+\left(\left(1-\beta_{n}\right) I-\alpha_{n} \mu F\right) S\left(\frac{x_{n}+x_{n+1}}{2}\right), \forall n \geqslant 0,
$$

where the parameters $\left\{\alpha_{n}\right\},\left\{\beta_{n}\right\}$ satisfy the conditions (C1)-(C3) of Theorem 3.1. Then the sequence $\left\{x_{n}\right\}$ converges strongly in $\mathrm{L}^{2}[0,1]$ to the solution of integral equation (4.5).

\subsection{Application to periodic solution of a nonlinear evolution equation}

Browder [5] proved the existence of a periodic solution of the time-dependent nonlinear evolution equation in a (real) Hilbert space $H$,

$$
\frac{d u}{d t}+A(t) u=f(t, u), t>0,
$$

where $A(t)$ is a family of closed linear operators in $H$ and $f: R \times H \rightarrow H$ satisfies the following conditions

(B1) $A(t)$ and $f(t, u)$ are periodic in $t$ of period $\xi>0$;

(B2) for each $t$ and each pair $u, v \in H$,

$$
\langle f(t, u)-f(t, v), u-v\rangle \leqslant 0 ;
$$

(B3) for each $t$ and each $u \in D(A(t)),\langle A(t) u, u\rangle \geqslant 0$;

(B4) there exists a mild solution $u$ of (4.6) on $R^{+}$for each initial value $v \in H$. Recall that $u$ is a mild solution of (4.6) with initial value $u(0)=v$ if, for each $t>0$,

$$
u(t)=u(t, 0) v+\int_{0}^{t} u(t, s) f(s, u(s)) d s,
$$

where $\{\mathrm{U}(\mathrm{t}, \mathrm{s})\}_{\mathrm{t} \geqslant s \geqslant 0}$ is the evolution system for the homogeneous linear system

$$
\frac{d u}{d t}+A(t) u=0, \quad(t>s)
$$

(B5) there exists some $\mathrm{R}>0$ such that

$$
\langle f(t, u), u\rangle<0
$$

for $\|u\|=R$ and all $t \in[0, \xi]$.

Note that under the conditions (B1)-(B5), the solution $u$ has period $\xi$ and $\|u(0)\|<R$.

We now apply our viscosity technique for IMR to (4.6). To this end, we define a mapping $\mathrm{T}: \mathrm{H} \rightarrow \mathrm{H}$ by

$$
\mathrm{T} v:=u(\xi), v \in \mathrm{H},
$$

where $u$ is the solution of (4.6) satisfying the initial condition $u(0)=v$. 
It is easy to find that $\mathrm{T}$ is nonexpansive. Moreover, the assumption (B5) implies that $\mathrm{T}$ is a self-mapping of the closed ball $\mathrm{B}:=\{v \in \mathrm{H}:\|v\| \leqslant \mathrm{R}\}$. Consequently, T has a fixed point in B which we denoted by $v$, and the corresponding solution $u$ of (4.6) is the periodic solution of (4.6) with periodic solution $\xi$ with the initial condition $u(0)=v$. In other words, finding a periodic solution of (4.6) is equivalent to find a fixed point of T. Therefore, our viscosity technique for IMR is applicable to (4.6). It turns out that the sequence $\left\{v_{n}\right\}$ defined by the IMR

$$
x_{n+1}=\alpha_{n} \rho U\left(x_{n}\right)+\beta_{n} x_{n}+\left(\left(1-\beta_{n}\right) I-\alpha_{n} \mu F\right) T\left(\frac{x_{n}+x_{n+1}}{2}\right), \forall n \geqslant 0,
$$

with the parameters $\left\{\alpha_{n}\right\},\left\{\beta_{n}\right\}$ satisfy the conditions (C1)-(C3) of Theorem 3.1, converges weakly to a fixed point $v$ of $T$, and then the corresponding mild solution $u$ of (4.6) with initial value $u(0)=\xi$ is a periodic solution of (4.6). Note that the iteration procedure (4.7) is essential to find a mild solution of the nonlinear evolution system (4.6) with the initial value $\left(v_{n}+v_{n+1}\right) / 2$.

\section{Conclusion}

The present work has been aimed to study the more general viscosity implicit midpoint rule for nonexpansive mapping and proved the strong convergence theorem for solving fixed point for a nonexpansive mapping. Theorem 3.1 extends and generalizes the general viscosity implicit midpoint rule of Rizvi [13], viscosity implicit midpoint rule of $\mathrm{Xu}$ et al. [19], and implicit midpoint rule of Alghamdi et al. [1] to a more general viscosity implicit midpoint rule for a nonexpansive mapping, which also includes the results of $[11,12,17]$ as special cases.

\section{Acknowledgment}

The author thanks Prof. Huaiping Zhu for hosting our visit in his Lamps Lab at York University where the research was completed.

The author would like to thank the editor and referees for useful comments and suggestions.

The author is supported by the research project of Shanghai Normal University (A-0231-17-001010).

\section{References}

[1] M. A. Alghamdi, M. A. Alghamdi, N. Shahzad, H.-K. Xu, The implicit midpoint rule for nonexpansive mappings, Fixed Point Theory. Appl., 2014 (2014), 9 pages. 1, 1, 1.1, 1, 3, 3.5, 4.4

[2] H. Attouch, Viscosity solutions of minimization problems, SIAM J. Optim., 6 (1996), 769-806. 1

[3] W. Auzinger, R. Frank, Asymptotic error expansions for stiff equations: an analysis for the implicit midpoint and trapezoidal rules in the strongly stiff case, Numer. Math., 56 (1989), 469-499. 1

[4] G. Bader, P. Deuflhard, A semi-implicit mid-point rule for stiff systems of ordinary differential equations, Numer. Math., 41 (1983), 373-398. 1

[5] F. E. Browder, Existence of periodic solutions for nonlinear equations of evolution, Proc. Nat. Acad. Sci. U.S.A., 53 (1965), 1100-1103. 2.6, 2.7, 4.4

[6] F. E. Browder, Convergence of approximants to fixed points of nonexpansive non-linear mappings in Banach spaces, Arch. Rational Mech. Anal., 24 (1967), 82-90. 1

[7] A. Cabot, Proximal point algorithm controlled by a slowly vanishing term: applications to hierarchical minimization, SIAM J. Optim., 15 (2005), 555-572. 4.2

[8] P. Deuflhard, Recent progress in extrapolation methods for ordinary differential equations, SIAM Rev., 27 (1985), 505-535. 1

[9] B. Halpern, Fixed points of nonexpanding maps, Bull. Amer. Math. Soc., 73 (1967), 957-961. 1

[10] P.-L. Lions, Approximation de points fixes de contractions, (French) C. R. Acad. Sci. Paris Sér. A-B, 284 (1977), A1357A1359. 2.6, 2.7, 4.3

[11] G. Marino, H.-K. Xu, A general iterative method for nonexpansive mappings in Hilbert spaces, J. Math. Anal. Appl., 318 (2006), 43-52. 1, 1.1, 1, 3.5, 4.4

[12] A. Moudafi, Viscosity approximation methods for fixed-points problems, J. Math. Anal. Appl., 241 (2000), 46-55. 1, 1, $1.1,3.5,4.4$ 
[13] S. H. Rizvi, General viscosity implicit midpoint rule for nonexpansive mapping, ArVix, 2016 (2016), 14 pages. 1, 1.1, 1, $3,3.5,4.4$

[14] C. Schneider, Analysis of the linearly implicit mid-point rule for differential-algebraic equations, Electron. Trans. Numer. Anal., 1 (1993), 1-10. 1

[15] S. Somalia, Implicit midpoint rule to the nonlinear degenerate boundary value problems, Int. J. Comput. Math., 79 (2002), 327-332.

[16] M. van Veldhuizen, Asymptotic expansions of the global error for the implicit midpoint rule (stiff case), Computing, 33 (1984), 185-192. 1

[17] H.-K. Xu, Viscosity approximation methods for nonexpansive mappings, J. Math. Anal. Appl., 298 (2004), 279-291. 1, $1.1,1,2.9,3.5,4.4$

[18] H.-K. Xu, Averaged mappings and the gradient-projection algorithm, J. Optim. Theory Appl., 150 (2011), 360-378. 4.1, 4.2

[19] H.-K. Xu, M. A. Alghamdi, N. Shahzad, The viscosity technique for the implicit midpoint rule of nonexpansive mappings in Hilbert spaces, Fixed Point Theory Appl., 2015 (2015), 12 pages. 1, 1.1, 1, 3, 3.5, 4.4

[20] I. Yamada, The hybrid steepest descent method for the variational inequality problem over the intersection of fixed point sets of nonexpansive mappings, Inherently parallel algorithms in feasibility and optimization and their applications, Haifa, (2000), Stud. Comput. Math., North-Holland, Amsterdam, 8 (2001), 473-504. 2.8 